\title{
Medical management of clomiphene-resistant polycystic ovarian syndrome: an update
}

\author{
Sharonjeet Kaur, Lekha Saha*
}

Department of Pharmacology, Postgraduate Institute of Medical Education and Research (PGIMER), Chandigarh-160012, India

Received: 26 October 2013 Accepted: 13 November 2013

\section{*Correspondence to:}

Dr. Lekha Saha,

Email: lekhasaha@rediffmail.com

\section{(C) 2014 Kaur $\mathrm{S}$ et al. This is an} open-access article distributed under the terms of the Creative Commons Attribution NonCommercial License, which permits unrestricted noncommercial use, distribution, and reproduction in any medium, provided the original work is properly cited.

\begin{abstract}
Clomiphene citrate is the traditional first-line treatment for chronic anovulation that characterizes polycystic ovary syndrome (PCOS). A gold standard therapy has always been Clomiphene Citrate (CC). However, 20\%$25 \%$ of PCOS women fail to ovulate with incremental doses of CC. A good body of evidence suggest that alternatives for PCOS women with CC-resistant anovulation include insulin sensitizers like metformin and pioglitazone. Insulin sensitizers improves pregnancy outcome and ovulation rate by and acts by ameliorating insulin sensitivity and hyperandrogenemia. Metformin is preferred in obese women. Gonadotropins induce ovulation and maintain optimal follicle growth via controlled administration of follicle stimulation hormone. Two regimens are used which includes high and low dose regimen. Low dose regimen is preferred but is associated adverse effects like ovarian hyperstimulation syndrome (OHSS) and increased cost. Extending clomiphene therapy reduces cost and improves pregnancy outcome. Glucocorticoids are preferably used when serum Dehydroepiandrosterone levels are $>200 \mu \mathrm{g} / \mathrm{dL}$. Bromocriptine improves ovulation rate by decreasing prolactin levels. Human Chorionic Gonadotropin restores ovulation but its use is limited during intrauterine insemination. Tamoxifen acts in a similar way as $\mathrm{CC}$ but has lesser antiestrogenic effect on the endometrium, cervical mucus, and granulosa cells, hence an added advantage of monofollicular ovulation. Aromatase inhibitors block conversion androstenedione and testosterone to estrogen in ovary and improves ovulation rate. Added advantage includes lesser cost, simple to use, no danger of multiple pregnancies and convenient for patient. Combination of $\mathrm{GnRH}$ analogues and Gonadotropins are associated with increased risk of OHSS. D-chiro-inositol, N-Acetylcysteine, melatonin and acarbose are tried with little success.
\end{abstract}

Keywords: PCOS, Infertility, Clomiphene citrate resistance, Drugs

\section{INTRODUCTION}

Poly cystic ovarian syndrome (PCOS), a commonest cause of an ovulatory infertility is a very heterogenous syndrome characterized by menstrual irregularities, manifesting as oligomenorrhea or amenorrhea, apart from abnormalities of hyperandrogenism and polycystic ovaries. It is considered to be the most prevalent endocrinopathy resulting in anovulation and affects 5$10 \%$ of women. ${ }^{1}$ The main pathophysiology of PCOS is hyperandrogenemia and insulin-resistance (IR). ${ }^{2,3}$ and in $70 \%$ of obese and $30 \%$ of thin PCOS patients, sententious insulin secretion is observed. ${ }^{4-6}$

The implication of the insulin resistance and compensatory hyperinsulinemia accelerate the effect of luteinizing hormone ( $\mathrm{LH})$ on ovarian theca cells and androgen synthesis which in turn inhibits ovulation through cessation of the follicular maturity process. Ghrelin is a growth hormone secretagogue and actively involved in regulation of weight and appetite. It has been found to be decreased in PCOS. It levels are highly correlated to the degree of insulin resistance, suggesting that ghrelin could be linked to insulin resistance in PCOS women. Vascular endothial growth factor regulates ovulation, corpus luteum and development of Ovarian Hyperstimulation Syndrome (OHSS). Women with PCOS have higher levels of VEGF and insulin promotes VEGF leading to OHSS. There are $40 \%$ of women suffering from PCOS that have to deal with infertility and abortion if pregnancy occurs in this group. The multitude of causes related to infertility in PCOS 
include oligo or anovulation, dysfunctional gonadotropin secretion, dysfunction of ovarian growth factors and proteins binding to them, high androgen levels, and insulin resistance.

\section{LITERATURE SEARCH}

To evaluate for different treatment modalities in PCOS, including the gold standard Clomiphene Citrate (CC) and $\mathrm{CC}$ resistant cases, we did electronic search through PubMed, Medline, Cochrane and focused on peerreviewed, full text, observational cohort or case control studies and randomized controlled trials using the keywords "Clomiphene Citrate", "resistant", "PCOS", "infertility". We excluded studies that were experimental or laboratory based. We also scanned all references from identified articles for additional relevant informations. There was no time, language or publication limit in our literature search. Various interventions have been proposed ranging from lifestyle modifications and administration of pharmaceutical agents such as CC, insulin-sensitizing agents, gonadotropins, and gonadotropin-releasing hormone $(\mathrm{GnRH})$ analogues, tamoxifen, to the use of laparoscopic ovarian drilling and the application of Assisted Reproduction Techniques (ART) like in vitro fertilization.

\section{LIFESTYLE MODIFICATIONS}

Multiple observational studies have reported positive results with weight loss regarding ovulation rates, which occurred spontaneously in women with $\operatorname{PCOS}^{7,8}$ and pregnancies have been reported after losing as little as $5 \%$ of initial body weight ${ }^{9}$ The treatment of obesity is multifaceted and involves behavioral counseling, lifestyle therapy (diet and exercise), pharmacologic treatment and bariatric surgery. ${ }^{10}$ Moreover, obese PCOS requires higher doses of $\mathrm{CC}$ and gonadotrophins. ${ }^{11}$ Routine exercise improves insulin sensitivity, reduce waist circumference, regularize menstrual cycle and promote weight loss leading to improved clinical outcome in PCOS women.

\section{CLOMIPHENE CITRATE (CC) AND RESISTANCE}

Classically $\mathrm{CC}$ is the first line approach to induce ovulation in patients with PCOS. It is a triphenylethylene derivative with estrogenic properties, but mainly acts as an anti-estrogenic. It constitutes an unequal mixture of two isomers, enclomiphene and zuclomiphene as their citrate salts, and prevents the action of the endogenous estrogen on the estrogenic receptors at the hypothalamicpituitary system. This blocks the ovarian negative feedback effect on gonadotrophin secretion, which results in increase in secretion of Follicle Stimulating Hormone (FSH) and Leutinizing Hormone (LH) leading to follicle development. $^{12}$

The cost of the medication is low, the oral route of administration is patient friendly, there are relatively few adverse effects, little ovarian response monitoring is required, and abundant clinical data are available regarding safety of the drug. ${ }^{13}$

The starting dose of CC generally should be $50 \mathrm{mg} /$ day (for 5 days, starting on days 2 to 5 after a spontaneous or progestin-induced withdrawal bleeding). The recommended maximum dose is $150 \mathrm{mg} /$ day as there is no clear evidence of efficacy at higher doses and this is in accord with Food and Drug Administration (FDA) recommendations of $750 \mathrm{mg}$ per treatment cycle ${ }^{13}$ for six (ovulatory) cycles. One of the side effects of CC therapy is propensity for multiple pregnancies, apart from vasomotor flushes and other risks like spontaneous abortion and Ovarian Hyper Stimulation Syndrome (OHSS). Although ovulation is observed in the $70-80 \%$ of PCOS women, only $40 \%$ of the PCOS women become pregnant.

There has been an epidemiological evidence of association of uterine cancer with clomiphene citrate treated for infertility. A retrospective cohort study conducted on 8,431 United states women (1965-88) demonstrated clomiphene citrate may increase the risk of uterine cancer [Risk Ratio (RR) 1.79, 95\% Confidence Interval (CI) 0.9, 3.4)] and also increase risk with increasing dose of clomiphene citrate (RR 1.93, 95\% CI $0.9,4.0$ with $>900 \mathrm{mg}$ ), menstrual cycles of use (RR 2.16, $95 \%$ CI 0.9, 5.2 for $\geq 6$ cycles) and time elapsed since initial use (RR 2.50, 95\% CI 0.9, 7.2 for women followed for $\geq 20$ years. In nulligravida and obese (RR 3.49, 95\% CI 1.3, 9.3) and obese (RR 6.02, 95\% CI 1.5, 108.0). ${ }^{14}$

$\mathrm{CC}$ resistance, is a term which refers to persistence of anovulation after standard CC therapy. CC has been defined differently with most define $\mathrm{CC}$ resistance as a lack of ovulation after six cycles of $\mathrm{CC}$, and some define it as a lack of ovulation and normal luteal phase after 5 or more cycles of up to $200 \mathrm{mg}$ or $250 \mathrm{mg}$ of $\mathrm{CC}$ for 5 days. ${ }^{15} \mathrm{CC}$ resistance affects approximately $15 \%$ to $40 \%$ of women with PCOS and remain a major challenge in gynecologic endocrinology. Failure to achieve pregnancy might be due to adverse effects of $\mathrm{CC}$ on the endometrium like endometrial thinning because of its anti-estrogenic actions as well as negative consequence on the cervical mucus, or because of luteinizing hormone hypersecretion. All this can be attributed to long half-life of CC of ( 2 weeks), which accumulates in the body and affects the endometrium and cervical mucus quality and quantity.

\section{FACTORS INVOLVED IN CLOMIPHENE RESISTANCE}

Various factors affecting contributing to resistance are hyperandrogenaemia, obesity and insulin resistance.

\section{DIFFERENT DRUG THERAPIES EVALUATED IN CC RESISTANT PCOS ARE AS FOLLOWS}




\section{Insulin sensitizing agents}

50 to $70 \%$ of PCOS women presents with insulin resistance. ${ }^{16}$ The presence of insulin resistance with compensatory hyperinsulinemia increases intraovarian androgen production, alters gonadotropin secretion or directly affects follicular development. The antidiabetic agent, metformin, has the advantage of increasing serum sex hormone-binding globulin (SHBG) levels and decreasing free androgen levels. It has been reported that metformin can improve pregnancy rates (PR) by improving the induction rate of ovulation. During the first week of usage it is associated with nausea and diarrhea which resolves after one week and rarely lactic acidosis. Metformin has a considerable beneficial role in obese PCOS. ${ }^{17}$ In a randomized control trial by Batukan et al. $4.2 \%$ of patients got pregnant at the end of CC cycles and $65.2 \%$ of the remaining group got pregnant with metformin plus CC cycles $(\mathrm{p}=0.0001) .{ }^{18}$ Heard et al. reported that, metformin alone in patients with PCOS results in a substantial number of pregnancies, with $69 \%$ $(20 / 29)$ of those who ovulated on receiving in less than 6 months. ${ }^{19}$ In a randomized, double blind study by Vandermolen et al. concluded that "metformin therapy significantly improved ovulatory rates and pregnancy rates as compared to placebo therapy in ovulatory PCOS women who are resistant to $\mathrm{CC}$ ". ${ }^{20}$ A 10 fold reduction in gestational diabetes ( $31 \%$ to $3 \%$ ) was found on use of metformin by Glueck CJ et al. ${ }^{21}$ Legro RS et al. randomly assigned 626 infertile women with the polycystic ovary syndrome to receive clomiphene citrate plus placebo, extended-release metformin plus placebo, or a combination of metformin and clomiphene for up to 6 months. Results depicted the live-birth rate was $22.5 \%$ (47 of 209 subjects) in the clomiphene group, $7.2 \%$ of 208) in the metformin group, and 26.8\% (56 of 209) in the combination therapy group $(\mathrm{P}<0.001$ for metformin vs. both clomiphene and combination therapy) $\mathrm{P}=0.31$ for clomiphene vs. combination therapy). Among pregnancies, the rate of multiple pregnancies was $6.0 \%$ in the clomiphene group, $0 \%$ in the metformin group, and $3.1 \%$ in the combination-therapy group. The rates of firsttrimester pregnancy loss did not differ significantly among the groups. However, the conception rate among subjects who ovulated was significantly lower in the metformin group $(21.7 \%)$ than in either the clomiphene group $(39.5 \%, \mathrm{P}=0.002)$ or the combination therapy group (46.0\%, $\mathrm{P}<0.001)$. With the exception of pregnancy complications, adverse-event rates were similar in all groups, though gastrointestinal side effects were more frequent and vasomotor and ovulatory symptoms less frequent, in the metformin group than in the clomiphene group. ${ }^{22}$ A comparative effectiveness trial was conducted comparing clomiphene citrate plus metformin and clomiphene citrate plus placebo in women with newly diagnosed polycystic ovary syndrome in randomized controlled multicentric trial on 228 women with polycystic ovary syndrome. The primary outcome measure was ovulation and secondary outcome measures were ongoing pregnancy, spontaneous abortion, and clomiphene resistance. The ovulation rate in the metformin group was $64 \%$ compared with $72 \%$ in the placebo group, a non-significant difference (Risk difference $-8 \%, 95 \%$ confidence interval $-20 \%$ to $4 \%$ ). There were no significant differences in either rate of ongoing pregnancy $40 \%$ [(CC + Metformin) versus $46 \%$ $(\mathrm{CC}+\mathrm{Placebo})]-6 \%$ (risk difference), $-20 \%$ to $7 \%$ $(95 \% \mathrm{CI})$ or rate of spontaneous abortion $12 \%[(\mathrm{CC}+$ Metformin) versus $11 \%(\mathrm{CC}+$ Placebo)] $1 \%$ (risk difference), $-7 \%$ to $10 \%$ (CI). A significantly larger proportion of women in the metformin group discontinued treatment because of side effects $16 \%$ [(CC + Metformin) versus 5\% (CC + Placebo)] 11\% (risk difference), $5 \%$ to $16 \%(\mathrm{CI})$. So to conclude metformin is not an effective addition to clomiphene citrate as the primary method of inducing ovulation in women with polycystic ovary syndrome. ${ }^{23}$ The primary risk with metformin is lactic acidosis, which is only seen in highrisk patients with renal, liver, or congestive heart failure.

Another insulin-sensitizing agent, pioglitazone, has been reported to be effective in metformin resistant PCOS and improves the ovulation rate. Several studies on pioglitazone use in PCOS patients resistant to metformin treatment or naive to any medication have appeared. A randomized controlled study compared the effectiveness of pioglitazone and metformin in fifty two PCOS patients suggested that pioglitazone was as effective as metformin in improving insulin sensitivity, hyperandrogenism, and pregnancy despite an increase in body mass index and waist-to-hip ratio. ${ }^{24}$ Another study demonstrated the effectiveness of pioglitazone in resistant PCOS patients with occurrence of successful pregnancies among seven of the nine patients. Among these seven, four of them delivered successfully. ${ }^{25}$ A randomized controlled trial of the efficacy of rosiglitazone and $\mathrm{CC}$ versus metformin and $\mathrm{CC}$ in women with CC-resistant polycystic ovary syndrome reported that short-term use of rosiglitazone and $\mathrm{CC}$ is more efficacious than metformin and $\mathrm{CC}$ in ovulation induction in women with CC-resistant PCOS. ${ }^{26}$

With regard to the use of these agents during pregnancy, metformin is a category $\mathrm{B}$ drug and pioglitazone and rosiglitazone are category $\mathrm{C}$ drugs, hence caution advised during the pregnancy.

\section{CC and gonadotropins}

Gonadotropins acts by inducing ovulation, maintaining and provoking optimal follicle growth via controlled administration of follicle stimulation hormone. Advantage against $\mathrm{CC}$ is that they do not exert peripheral antioestrogenic effect. Risk of ovarian hyperstimulation syndrome and multiple pregnancies is a main drawback, apart from requiring an expertise to administer it. OHSS is characterized by a shift of fluid from the intravascular space into the abdominal cavity creating a massive third space. The procedure is time consuming and therapy is expensive. ${ }^{27}$ Subsequent efforts to reduce the frequency of 
ovarian hyperstimulation have resulted in the development of low-dose protocols (37.5-75 IU/day)

\section{Currently, two low-dose regimens are used}

- $\quad$ Step up regimen Step-up regimens are based upon the principle of a stepwise increase in FSH supply to determine the FSH threshold for follicular development.

- Step down regimen This regimen is designed to achieve the FSH threshold through a loading dose of FSH with a subsequent stepwise reduction as soon as follicular development is observed on ultrasound

American Society for Reproductive Medicine (ASRM) recommends low dose gonadotropins protocol. ${ }^{28}$

A step up regimen constitutes starting up with minimum dose (37.5-50 IU) and increasing accordingly to lack of follicle response. If no follicle is observed by 1 week, during evaluation with ultrasound, then dose is increased by $50 \%$ each time.

Step down regimen starts with maximum recommended dose which is decreased as a follicle response is achieved. In this case also a $50 \%$ decrease in dose each time is done as the regimen is changed. A maximum of six cycles of gonadotropins is recommended because no response with six cycles signifies resistance. An advantage of low dose regimen is that it results in ovulation rate of $70 \%$, a pregnancy rate of $20 \%$ per cycle and multiple live birth rate of $5.7 \%$ while maintaining a low incidence of multiple pregnancies $(<6 \%)$ and $\operatorname{OHSS}(<1 \%) .^{29} \mathrm{~A}$ comparative study between CC (group A) versus CC plus Human menopausal Gonadotropin (Group 2) in conjugation with intrauterine insemination, in 117 infertile couples demonstrated pregnancy rate of $6.52 \%$ in group $\mathrm{A}$ and $12.21 \%$ in group $\mathrm{B}(\mathrm{p}=0.22)$ with no multiple gestation and obvious hyperstimulation syndrome. ${ }^{30}$ A Cochrane review comparing safety and effectiveness in terms of ovulation, pregnancy, miscarriage, multiple pregnancy rate and ovarian hyperstimulation syndrome (OHSS) of rFSH with uFSH and different dose regimens of $\mathrm{rFSH}$ reported. No significant differences were demonstrated for the relevant outcomes. The odds ratio for ovulation rate was 1.19 (95\% CI 0.78, 1.80), for pregnancy rate 0.95 (95\% CI $0.64,1.41)$, for miscarriage rate 1.26 (95\% CI $0.59,2.70)$, for multiple pregnancy rate $0.44(95 \% \mathrm{CI} 0.16,1.21)$ and for OHSS 1.55 (95\% CI 0.50, 4.84). Only randomised trial comparing chronic low dose versus conventional regimen (Gonadotropin) with rFollicle Stimulating Hormone $(\mathrm{rFSH})$ reported no significant differences among them. ${ }^{31}$

The incidence of multiple births after gonadotropin therapy is high ranging from $11-44 \%{ }^{32}$ It is further associated with preterm delivery because of hormone relaxin which can soften and ripen the uterine cervix.

\section{Extended clomiphene citrate treatment}

Extended clomiphene therapy we mean that the duration for which clomiphene is given is extended beyond 5 days. A retrospective observational analysis conducted on 24 anovulatory patients who failed on $150 \mathrm{mg}$ of CC administration for 5 days, by Isaacs et al. had demonstrated high rate of ovulation and pregnancy when $\mathrm{CC}$ was given daily starting on cycle days 3 of menses for 9 days (extended) at a starting dose of 100 to 150 $\mathrm{mg} / \mathrm{d}$, along with prednisone $5 \mathrm{mg}$ orally each night throughout the cycle in clomiphene resistant cases. ${ }^{33}$ This study offers an alternative before gonadotropins administration or surgery is done in terms of reduced cost and risk. A randomized controlled trial was conducted comparing $100 \mathrm{mg}$ of extended (day 2 to day 9) CC versus gonadotropin 75 IU intramuscular for 5 days started day 2 of menses and evaluated the outcomes on ovulation rate, follicle stimulation and pregnancy rate. ${ }^{34}$

The results comparing gonadotropin and extended CC therapy are as follows in Table 1.

Table 1: The results comparing gonadotropin and extended CC therapy.

\begin{tabular}{|llll|}
\hline Outcomes & Gonadotropin & $\begin{array}{l}\text { Extended } \\
\text { clomiphene } \\
\text { citrate }\end{array}$ & P value \\
\hline $\begin{array}{l}\text { Ovulation } \\
\text { rate }\end{array}$ & $57.6 \%$ & $28.1 \%$ & 0.001 \\
\hline $\begin{array}{l}\text { Follicle } \\
\text { stimulation }\end{array}$ & $6.7 \pm 0.3$ & $4.1 \pm 0.04$ & 0.01 \\
\hline $\begin{array}{l}\text { Pregnancy } \\
\text { rate }\end{array}$ & $20.2 \%$ & $11.4 \%$ & 0.03 \\
\hline
\end{tabular}

Extended therapy resulted in modest ovulation, pregnancy rate with no side effects and offers safety, efficacy and economic advantage.

\section{CC and glucocorticoids}

The concept behind adding glucocorticoids (dexamethasone or prednisone $\mathrm{mg}$ ) has shown to induce ovulation in CC resistant women. Glucocorticoids are used when serum DHEA-S concentration is $>200 \mu \mathrm{g} / \mathrm{dL}$. The therapy should be continued if ovulation is observed otherwise it should be discontinued. A prospective randomized placebo controlled study was conducted in 80 infertile women with $\mathrm{CC}$ resistant PCOS, comparing combination of CC (100mg/day) given from (day 3 to day 7 of cycles) and dexamethasone $2 \mathrm{mg} /$ day (From day 2 to day 12 cycle) versus CC plus placebo on ovulation (Primary outcome) and number of follicles $>18 \mathrm{~mm}$, endometrial thickness and pregnancy rates (Secondary outcome). Results depicted significantly higher rate of ovulation in $\mathrm{CC}$ plus dexamethasone group (75\% versus $15 \%)(\mathrm{p}<0.001)$ and pregnancy $(40 \%$ versus $5 \%)(\mathrm{p}<$ $0.05)$. The mean number of follicles $>18 \mathrm{~mm}$, mean 
endometrial thickness were significantly higher in $\mathrm{CC}$ plus dexamethasone group $(\mathrm{p}<0.05){ }^{35}$

\section{Bromocriptine}

The elevated levels of LH which are characteristic of the PCOS can be reduced by a dopamine infusion suggesting a relative dopamine deficiency in women with PCOS. Bromocriptine has therefore been proposed as an alternative method of ovulation induction. Studies have demonstrated that addition of bromocriptine to the CC treatment leads to decrease in prolactin level and improved ovulation. Bromocriptine in dose of $7.5 \mathrm{mg}$ up to 1 year with normal prolactin levels in 20 patients improved leutinizing hormone (LH), LH/FSH ratio and testosterone and also improves hirsuitsm and finally restoration of cyclical ovarian function and decrease androgen synthesis. A trial conducted in 43 women with PCOS with normal serum prolactin levels and clomiphene resistance reported bromocriptine administration along with CC lead to regular menstrual cycle in $40 \%$ of the patient and $30 \%$ conceived. It also decreased prolactin level from $269.9 \mathrm{mIU} / \mathrm{ml}$ to $174 \mathrm{mIU} / \mathrm{ml}$ ( $\mathrm{p}<0.001)$, $\mathrm{LH}$ from 17.9 to $11.5(\mathrm{p}<0.001)$ and $\mathrm{LH} / \mathrm{FSH}$ ratio from 2.4 to $1.5(\mathrm{p}<0.001) .^{36}$ This suggest restoration and improvement of the impaired hypothalamus - pituitary axis after bromocriptine administration. Another long acting ergoline D2 agonist, cabergoline, has shown a good evidence of ovarian response in hyperprolactinemic patients with PCOS candidates for treatment with gonadaotrophins. ${ }^{37}$

\section{Clomiphene and $h C G$}

The use of human Chorionic Gonadotropin (hCG) is limited during intrauterine insemination to trigger ovulation. Studies have been shown that intrauterine insemination (IUI) performed after exogenous hCGtriggered ovulation in $\mathrm{CC}$ induced cycles is no more effective than IUI performed after detection of the endogenous LH surge. ${ }^{38}$

\section{Anti-estrogens other than CC (Tamoxifen)}

Tamoxifen has a similar mechanism to $\mathrm{CC}$ but it has a much lesser antiestrogenic effect on the endometrium, cervical mucus, and granulosa cells, hence an added advantage of monofilliclar ovulation. ${ }^{39}$ Tamoxifen also does not increase follicular phase FSH and LH levels and leutal phase progesterone. So its postulated action is directly on ovary rather than through hypothalamic pituitary axis. Lack of response to the clomiphene treatment leads to more expensive and serious treatments like gonadotropins, IUI and in vitro fertilization (IVF). Treatment with gonadotropin places women at risk for ovarian hyperstimulation syndrome, multiple gestation, recurrent abortion, and high costs. ${ }^{40} \mathrm{~A}$ study comparing $\mathrm{CC}$ plus tamoxifen versus laproscopic ovarian drilling in $\mathrm{CC}$ resistant women with PCOS in 150 women in which primary outcome was live birth rate in each group and secondary outcome were rates of ovulation, clinical pregnancy and miscarriage. Results depicted no significant difference between groups regarding rates of ovulation $(81.3 \%$ versus $85.3 \%)$, pregnancy rate $(53.3 \%$ versus $50 \%)$ and an live birth rate $(49.3 \%$ versus $44 \%$ ) but mean endometrial thickness was significantly greater on day HCG in medication group $(\mathrm{p}<0.001) .{ }^{39}$ Hence this study suggest that $\mathrm{CC}$ plus tamoxifen was as effective laproscopic ovarian drilling in promoting ovulation and pregnancy. Similar results were reported in another study which reported 86 anovulatory women under 49 years, comparing CC with tamoxifen showed rate of ovulation in tamoxifen group $44.2 \%$ and in CC group $45.1 \%$. Pregnancy rate in tamoxifen citrate was 10 and 6 pregnancies in $\mathrm{CC}$ group. The fecundity per ovulatory cycle was $20.0 \%$ in tamoxifen group and $14.6 \%$ in CC group. ${ }^{41}$ According to the results of the study by Mohammad Ghafourzadeh et al. Tamoxifen + CC increases the ovulation rate only in age group of 25 to 39 years old with $20 \leq \mathrm{BMI} \leq 26.99$ women, and also reported that $\mathrm{CC}$ plus tamoxifen was as effective as laparoscopic ovarian drilling (LOD) in promoting ovulation and pregnancy. ${ }^{42}$ One metanalysis pooled results from 4 trials comparing tamoxifen with CC reported similar rate of ovulation among both the groups, [Odd Ratio (OR) $0.7555,95 \%$ CI (CI 0.513-1.111)] and pregnancy rates reported no added benefit (OR 1.056 , 95\% CI 0.58 $1.912) .^{43}$

\section{Aromatase inhibitors}

The concept of using Aromatase Inhibitors (AIs) has been extensively investigated by several research groups in the past few years. ${ }^{44-46}$ The proposed mechanism of action is blockage of the estrogen production by inhibiting aromatization which will further stops the conversion of androstenedione and testosterone to estrogen in ovary. Ovulation can be induced in the earlier follicular phase by aromatase inhibitors as they will cause the hypoestrogenic state, that would release the hypothalamus-pituitary axis from Estrogen (E) negative feedback on GnRH and gonadotropin secretion, which would stimulate ovarian follicular development. Further it may act locally in the ovary to increase follicular sensitivity to FSH by accumulation of intraovarian androgen. ${ }^{44}$ Aromatase inhibitors possess an additive advantage of having short half-life of $45 \mathrm{hrs}$ so estrogen target tissues (e.g., endometrium and cervix) are spared adverse effects. Its peripheral action increase follicular sensitivity to follicle stimulating hormone through amplification of $\mathrm{FSH}$ receptor gene expression. Aromatase inhibitors have the advantage of less side-effects on endometrial thickness than has CC, and possibly a lower risk of multiple pregnancies, due to lesser ovarian stimulation. ${ }^{34}$ However; fetal abnormalities are the possible concern related to aromatase inhibition. Multiple pregnancies did not occur.

The effectiveness of letrozole has been evaluated on hormonal levels and pregnancy rate. A total of 41 patients with unexplained infertility undergoing 
intrauterine insemination therapy were randomized to receive either letrozole $+\mathrm{rFSH}$ or $\mathrm{CC}+\mathrm{rFSH}$. Results depicted a significantly lower peak serum E2 in letrozole $+\mathrm{rFSH}$ as compared to CC + rFSH $(914+/-187$ vs 1207 $+/-309 \mathrm{pg} / \mathrm{ml}$ ). There was difference in number of mature preovulatory follicles $(>16 \mathrm{~mm})$ and clinical pregnancy rate between two groups (23.8\% versus $20 \%)$. However, higher endometrial thickness was observed in letrozole group at the time of $\mathrm{HcG}$ administration $(9.5+/-1.5 \mathrm{~mm}$ versus $7.3+/-1.1 \mathrm{~mm}, \mathrm{p}<0.000){ }^{45}$ Another comparative study between letrozole versus HMG in IUI in 80 women found no difference in clinical pregnancy rate (18.4 versus $15.7 \%$ ). Advantage found was its cost, simplicity and convenience for patients. ${ }^{46}$ Letrozole as compared to anastrozole in CC resistant women showed no difference in ovulation rate $(62 \%$ versus $63.4 \%)$ and pregnancy rate was $(12.2 \%$ in letrozole versus $15.1 \%$ in anastrazole $) .{ }^{44}$

\section{Combination of GnRH analogues and gonadotropins}

The concomitant use of a GnRH agonist with gonadotropin administration to improve pregnancy rates in patients undergoing ovulation induction has not been firmly established. ${ }^{44}$ Moreover; combined therapy was associated with an increased risk of OHSS. Therefore, the significantly higher hyperstimulation rate, the associated risk of multiple pregnancies, and the additional inconvenience and cost of concomitant GnRH agonist administration, in the absence of documented increases in pregnancy success, creates a dilemma regarding its use and does not justify the routine use of GnRH agonists during ovulation induction with gonadotropins in PCOS patients. $^{44}$

\section{D-Chiro-inositol}

Some of the actions of insulin may involve low molecular-weight inositol phosphoglycan mediators. When insulin binds to its receptor, mediators of this class are generated by hydrolysis of glycosylphosphatidylinositol lipids located at the outer leaflet of the cell membrane. Although different species have been identified, an inositol phosphoglycan molecule containing D-chiro-inositol and galactosamine is known to have a role in activating key enzymes that control the oxidative and nonoxidative metabolism of glucose.

A deficiency of the D-chiro-inositol phosphoglycan, a mediator of the action of insulin may result in resistance to insulin. Insulin resistance has been linked to decreased urinary excretion of chiro-inositol (a component of the putative D-chiro-inositol phosphoglycan mediator) in primates $^{47}$ and in humans with impaired glucose tolerance. $^{48}$ The amount of chiro-inositol in muscle is lower in subjects with type 2 diabetes mellitus than in normal subjects Administration of D-Chiro-insitol has been demonstrated to improve glucose tolerance, decrease serum androgens and improve ovulation in PCOS patients. ${ }^{49}$ Nestler $\mathrm{J}$ E reported improvement in the insulin sensitivity by D-chiro-inositol hence improves ovulatory function and decreases serum androgen concentrations along with blood pressure and metabolic parameters such as plasma triglyceride concentrations. ${ }^{50}$

\section{N-Acetylcysteine}

$\mathrm{N}$-acetyl cysteine (NAC), an antioxidant and a mucolytic is the acetylated precursor of both the amino acid Lcysteine and reduced glutathione. It has been proven to have activity on insulin secretion in pancreatic cells, as well as on the regulation of the insulin receptor in human erythrocytes.

Studies have illustrated that PCOS patients with hyperinsulinemia, treated with NAC resulted in improvement of insulin sensitivity and a significant decline in plasma testosterone and lipid levels. A study reported combination of clomifene citrate (CC) and NAC increases ovulation and pregnancy rates in CC-resistant PCOS patients. ${ }^{51}$ Taking its lack of adverse effects into consideration, NAC can be regarded as an appropriate substitute for insulin-reducing medications in the treatment of PCOS patients.

\section{Melatonin}

Melatonin has been shown to have direct effects on ovarian function by acting as a powerful antioxidant and receptor independent free radical scavenger, as reactive oxygen species and apoptosis are involved in a number of reproductive events which includes folliculogenesis, follicular atresia, ovulation, oocyte maturation, and corpus luteum (CL) formation. A substantial amount of scientific evidence supports a local role of melatonin in the human reproductive processes, hence its role in pathophysiology of PCOS, endometriosis and premature ovarian failure. ${ }^{52}$

\section{Acarbose}

Acarbose is used in type 2 diabetes, reduces the postprandial rise in both serum glucose and insulin levels by inhibiting $\alpha$-glucosidase, an enzyme responsible for the intestinal absorption of carbohydrates.

In patients with PCOS, it has been demonstrated that acarbose therapy improves various clinical manifestations of PCOS which includes insulin resistance, signs and symptoms of hyperandrogenism, menstrual irregularity, obesity (in women with BMI $>30 \mathrm{~kg} / \mathrm{m}^{2}$ ), and infertility. ${ }^{53}$ To date, acarbose has been compared with only metformin in terms of hormone concentrations, weight loss, menstrual regularity, and ovulation rates The most widely reported adverse effect of treatment with acarbose was related to gastrointestinal adverse effects. Although the findings from all these trials described are extremely promising, none assessed medication adherence, which is important with a drug typically taken three times a day. 


\section{DISCUSSION AND CONCLUSION}

$\mathrm{CC}$ is still the typical therapy used for inducing ovulation in this condition, still emphasis should also on lifestyle modification like weight loss and exercise. It is not, however, equally successful in all situations for different reasons. Alternatives for PCOS women with CC-resistant anovulation includes, insulin sensitizing agents which are preferred in hyperinsulinemic state and metformin in obese women. Combination of GnRH analogues and gonadotropins is a costly option along with risk of OHSS, extending $\mathrm{CC}$ treatment is cheap alternative with similar efficacy as CC, aromatase inhibitors improves ovulation rate with no risk of multiple pregnancies, tamoxifen restores ovulation and has a lesser adverse effects as compared to $\mathrm{CC}$, bromocriptine induces ovulation by decreasing prolactin levels and normalizes hypothalamic pituitary axis, clomiphene and glucocorticoids are used when DHAE levels are $>200 \mu \mathrm{g} / \mathrm{dl}$. D-chiro-inositol, Nacetylcysteine, melatonin and acarbose are being tried with little success. Last but not the least is another methodology called in vitro fertilization which remains another effective line of treatment of CC resistant PCOS, with the advantage of reduced multiple pregnancies and tried in those with tubal damage, severe endometriosis, preimplantation genetic diagnosis and male factor infertility.

All these regimens have distinct therapeutic efficacy, added advantages and drawbacks and therapy should be instituted based on individual case requirement.

Funding: No funding sources Conflict of interest: None declared

Ethical approval: Not required

\section{REFERENCES}

1. Balen A. H., The current understanding of polycystic ovary syndrome. Obstet Gynaecol. 2004;6:66-74.

2. Diamanti-Kandarkis E. Polycystic ovarian syndrome pathophysiology, molecular aspects and clinical implications. Expert Rev Mol Med. 2008;10:1-21.

3. Schuring AN., Schulte N., Sonntag B., Kiesel L. Androgens and insulin-two key players in polycystic ovary syndrome. Recent concepts in the pathophysiology and genetics of polycystic ovary syndrome. Gynakol Geburtshilfliche Rundsch. 2008;48:9-15.

4. Fulghesu AM., Ciampelli M., Muzj G., Belosi C., Selvaggi L., Ayala GF., Lanzone A.N-acetylcysteine treatment improves insulin sensitivity in women with polycystic ovary syndrome. Fertil Steri. 2002;77:1128-35.

5. Randeva HS, Lewandowski KC, Drzewoski J, Brooke-Wavell K, O'Callaghan C, Czupryniak L, Hillhouse EW, Prelevic GM. Exercise decreases plasma total homocysteine in overweight young women with polycystic ovary syndrome. J Clin Endocrinol Metab. 2002;87:4496-4501.
6. Ketel IJ., Stehouwer CD., Serné EH., Korsen TJ., Hompes PG., Smulders YM., de Jongh RT., Homburg R., Lambalk CB.. Obese but not normal weight women with polycystic ovary syndrome are characterized by metabolic and microvascular insulin resistance. J Clin Endocrinol Metab. 2008;93:3365-72.

7. Pasquali R., Pelusi C., Genghini S., Cacciari M., Gambineri A. Obesity and reproductive disorders in women. Hum Reprod Update. 2003;9:359-72.

8. Moran LJ., Noakes M., Clifton PM., Tomlinson L., Galletly C., Norman RJ. Dietary composition in restoring reproductive and metabolic physiology in overweight women with polycystic ovary syndrome. J Clin Endocrinol Metab. 2003;88:812-9.

9. Kiddy DS., Hamilton-Fairley D., Bush A., Short F., Anyaoku V., Reed MJ., Franks S. Improvement in endocrine and ovarian function during dietary treatment of obese women with polycystic ovary syndrome. Clin Endocrinol. 1992;36:105-11.

10. Yanovski S Z and Yanovski JA. Obesity. N Engl J Med. 2002;346:591-602.

11. Norman RJ., Davies MJ., Lord J. \& Moran LJ. The role of lifestyle modification in polycystic ovary syndrome. Trends in Endocrinology and Metabolism. 2002Aug;13(6):251-7.

12. The Thessaloniki ESHRE/ASRM-Sponsored PCOS Consensus Workshop Group. Consensus on infertility treatment related to polycystic ovary syndrome. Fertil Steril. 2008;89:505-22.

13. Dickey RP., Taylor SN., Curole DN., Rye PH., Pyrzak R. Incidence of spontaneous abortion in clomiphene pregnancies. Hum Reprod. 1996Dec;11(12):2623-8.

14. Westhoff CL., Scoccia B., Lamb EL., Lubin JH., Brinton LA. Uterine Cancer after Use of Clomiphene Citrate to Induce Ovulation. Am $\mathrm{J}$ Epidemiol. 2005;161:607-15.

15. Zakherah M S., Nasr A., Saman A M E., Shaaban O M., Shahin A Y. CC plus tamoxifen versus laparoscopic ovarian drilling in women with clomiphene-resistant polycystic ovary syndrome. Int J Gynaecol Obstet. 2010;108:240-3.

16. Legro, RS Castracane, VD. \& Kauffman, RP. Detecting insulin resistance in polycystic ovary syndrome purposes and pitfalls. Obstetrical and Gynecological Survey. 2004;59:141-54.

17. Kowalska I., Kinalski M., Straczkowski M., Wolczyski S., Kinalska I. Insulin, leptin, IGF-I and insulin-dependent protein concentrations after insulin-sensitizing therapy in obese women with polycystic ovary syndrome. Eur J Endocrinol. 2001;144:509-15.

18. Batukan C., Baysal B., Metformin improves ovulation and pregnancy rates in patients with polycystic ovary syndrome. Arch Gynecol Obstet. 2001;265:124-7.

19. Heard M J., Pierce A., Carson SA., Buster JE. Pregnancies following use of metformin for 
ovulation induction in patients with polycystic ovary syndrome. Fertil Steril. 2002;77:669-73.

20. Vandermolen DT., Ratts VS., Evans WS. Metformin increases the ovulatory rate and pregnancy rate from clomiphene citrate in patients with polycystic ovary syndrome who are resistant to clomiphene citrate alone. Fertil Steril. 2001;752:310-5.

21. Glueck CJ., Wang P., Kobayashi S., Phillips H., Sieve-Smith L. Metformin therapy throughout pregnancy reduces the development of gestational diabetes in women with polycystic ovary syndrome. Fertil Steril. 2002;77:520-5.

22. Legro RS., Barnhart HX., Schlaff WD., Carr BR., Diamond MP. , Carson SA. Clomiphene, metformin, or both for infertility in the polycystic ovary syndrome. N Engl J Med. 2007;356:551- 66.

23. Moll E., Bossuyt PM., Korevaar JC., Lambalk CB., Van D V. Effect of clomifene citrate plus metformin and clomifene citrate plus placebo on induction of ovulation in women with newly diagnosed polycystic ovary syndrome randomised double blind clinical trial. BMJ. 2006;332:1485-90.

24. Ortega-Gonzalez C., Luna S., Hernandez L., Crespo G., Aguayo P., Arteaga- Troncoso G. Responses of serum androgen and insulin resistance to metformin and pioglitazone in obese, insulin-resistant women with polycystic ovary syndrome. J Clin Endocrinol Metab. 2005;90:1360-5.

25. Ota H., Goto T., Yosioka T., Ohyama N. Successful pregnancies treated with pioglitazone in infertile patients with polycystic ovary syndrome. Fertil Steril. 2008;90:709-1.

26. Rouzi AA, Ardawi MS. A randomized controlled trial of the efficacy of rosiglitazone and $\mathrm{CC}$ versus metformin and $\mathrm{CC}$ in women with $\mathrm{CC}$-resistant polycystic ovary syndrome. Fertil Steril. 2008;85:428-35.

27. Badaway A., Elanshar A. Treatment options for PCOS syndrome. Int J Womens Health. 2001;3:2535.

28. Christin-Maitre S., Hugues J N. Recominary FSH study group. A comparative randomized multicentric study comparing the step up versus step down protocol in polycystic ovary syndrome. Hum Reprod. 2003;18:1626-31.

29. Homburg R, Howles CM. Low dose FSH therapy for anovulatory infertility associated with polycystic ovary syndrome rational, results, reflections and refinements. Hum repro update. 1999;5:493-9.

30. Rashidi BH., Gharai M., Momeni M., Tehraninejad ES. A comparision of $\mathrm{CC}$ and sequential $\mathrm{CC}$ plus human menopausal gondaotropins for use in conjugation with intrauterine insemination. Acta Medica Iranicaa. 2005;433:187-92.

31. Bayram N., Van Wely M., Van der Veen F. Recombinant FSH versus urinary gonadotrophins or recombinant FSH for ovulation induction in subfertility associated with polycystic ovary syndrome. Cochrane Database of Systematic
Reviews. 2001;2:CD002121. DOI 10.1002/14651858.CD002121.

32. Spadoni, L., R, Cox, W., and Smith. C. Use of human Menopausal Gonadotropin for the induction of ovulation. Am J Obstet Gynecol. 1986;120:9881001.

33. Isaacs JD., Lincoln SR., Cowan BD. Extended CC $\mathrm{CC}$ and prednisone for the treatment of chronic anovulation resistant to $\mathrm{CC}$ alone. Fertil Steril. 1997;67:641-3.

34. Badawy A., Allam A., Abulatta M. Extending clomiphene treatment in clomiphene-resistant women with PCOS a randomized controlled trial. Reprod Biomed Online. 2008;16: 825-9.

35. Elnashar A., Abdelmageed E., Fayed M., Sharaf M. $\mathrm{CC}$ and dexamethasone in treatment of CC-resistant polycystic ovary syndrome a prospective placebocontrolled study. Hum reprod. 2006;21:1805-8.

36. Parsanezhad ME., Alborzi S., Rosamsoolat S., Kokabi E. Effect of bromocriptine in polycystic ovarian syndrome with clomiphene citrate resistant. Journal of reproduction and infertility. 2000;1:4-11.

37. Papaleo, E. Doldi, N. De Santis, L. Marelli, G. Marsiglio, E. Rofena, S. \& Ferrari, A. Cabergoline influences ovarian stimulation in hyperprolactinaemic patients with polycystic ovary syndrome. Human Reproduction. 2001;16:2263-66.

38. Deaton JL., Clark RR., Pittaway DE., Herbst P., Bauguess P. CC ovulation induction in combination with a timed intrauterine insemination the value of urinary luteinizing hormone versus human chorionic gonadotropin timing. Fertil Steril. 1997;68:43-7.

39. Zakherah M S., Nasr A., Saman A M E., Shaaban O M., Shahin A Y. CC plus tamoxifen versus laparoscopic ovarian drilling in women with clomiphene-resistant polycystic ovary syndrome. Int J Gynaecol Obstet. 2010;108:240-3.

40. Shahin AY. The problem of IVF cost in developing countries has natural cycle IVF a place? Reprod Biomed Online. 2007;25:1551-6.

41. Boost Anfor R., Jain JK., Mishell DR., Paulson RJ. A prospective randomized trial comparing $\mathrm{CC}$ with tamoxifen citrate for ovulation induction. Fertil Steril. 2001;75:1024-6

42. Ghafourzadeh M., Karimi M., Karimazadeh M A., Bokai M. Comparison between Two Methods of Ovulation Induction Clomiphene alone and Clomiphene +Tamoxifen in PCOS Patients. IJRM. 2004;2:74-7.

43. Steiner AZ., Terplan M., Paulson RJ. Comparison of tamoxifen and $\mathrm{CC}$ for ovulation induction a metanalysis. Human Reproduction 2005; 1511-5

44. Casper RF and Mitwally MFM. Casper RF., Mitwally MFM. Casper RF., Mitwally MFM. Review Aromatase Inhibitors for Ovulation Induction. J Clin Endocrinol Metab. 2006;91:76071.

45. Barroso G., Menocal G., Felix H., Rojas-Ruiz JC., Arslan M., Oehninger S. Comparison of the efficacy of the aromatase inhibitor letrozole and $\mathrm{CC}$ as 
adjuvants to recombinant follicle-stimulating hormone in controlled ovarian hyperstimulation a prospective, randomized, blinded clinical trial. Fertil Steril. 2006;86:1428-31.

46. Baysoy A., Serdaroglu H., Jamal H., Karatekeli E., Ozornek H., Attar E. Letrozole versus human menopausal gonadotrophin in women undergoing intrauterine insemination. Reprod Biomed Online. 2006Aug;13(2):208-12.

47. Ortmeyer HK., Bodkin NL., Lilley K., Larner J., Hansen BC. Chiroinositol deficiency and insulin resistance. I. Urinary excretion rate of chiroinositol is directly associated with insulin resistance in spontaneously diabetic rhesus monkeys. Endocrinology. 1993;132:640-5.

48. Suzuki S, Kawasaki H, Satoh Y, Ohtomo M, Hirai M, Hirai A, Hirai S, Onoda M, Matsumoto $\mathrm{M}$, Hinokio Y. Urinary chiro-inositol excretion is an index marker of insulin sensitivity in Japanese type II diabetes. Diabetes Care. 1994;17:1465-8.

49. Nestler J., Jakubowicz J., and Iurono J. Role of inositophosphoglycan mediators of insulin action in the polycystic ovary syndrome. J Pediatr. Endocrinol Metab. 2000;13Suppl 5:1295-8.

50. Nestler JE., Jakubowicz DJ., Reamer P., Gunn RD., M.S., Allan G. Ovulatory and Metabolic Effects of D-Chiro-Inositol in the Polycystic Ovary Syndrome. New Engl J Med. 1999;340:1314-20.

51. R Rizk AY, Bedaiwy MA, Al-Inany HG. N-acetylcysteine is a novel adjuvant to $\mathrm{CC}$ in $\mathrm{CC}$-resistant patients with polycystic ovary syndrome. Fertil Steril. 2005;83:367-70.

52. Tamura H, Nakamura Y, Korkmaz A, Manchester LC, Tan DX, Sugino N, Reiter RJ. Melatonin and the ovary physiological and pathophysiological implications. Fertil Steril. 2009;92:328-43.

53. Sönmez AS, Yasar L, Savan K, Koc S, Ozcan J, Toklar A. Comparison of the effects of acarbose and metformin use on ovulation rates in CC-resistant polycystic ovary syndrome. Hum Reprod. 2005Jan;20(1):175-9.

doi:10.5455/2319-2003.ijbcp20140232

Cite this article as: Kaur S, Saha L. Medical management of clomiphene-resistant polycystic ovarian syndrome: an update. Int J Basic Clin Pharmacol 2014;3:1-9. 